\title{
Detecting changes in the basin of attraction of a dynamical system: Application to the postural restoring system
}

\author{
Maria S. Zakynthinaki ${ }^{\mathrm{a}, *}$, Alfonso López ${ }^{\mathrm{b}}$, Carlos A. Cordente ${ }^{\mathrm{b}}$, Jonathan A. Ospina Betancurt ${ }^{\mathrm{b}}$, \\ Manuel Sillero Quintana ${ }^{b}$, Javier Sampedro ${ }^{b}$
}

a Applied Mathematics and Computers Laboratory, Department of Sciences, Technical University of Crete, 73100 Chania, Greece

${ }^{\mathrm{b}}$ Facultad de Ciencias de la Actividad Física y del Deporte, Universidad Politécnica de Madrid, Avda Martin Fierro 7, 28040 Madrid, Spain

\section{A R T I C L E I N F O}

\section{Keywords:}

Modelling

Curve fitting

Dynamical system

Human balance

\begin{abstract}
A B S T R A C T
A method that provides a three-dimensional representation of the basin of attraction of a dynamical system from experimental data was applied to the problem of dynamic balance restoration. The method is based on the density of the data on the phase space of the system under study and makes use of modeling and numerical curve fitting tools. For the dynamical system of balance restoration, the shape and the size of the basin of attraction depend on the dynamics of the postural restoring mechanisms and contain important information regarding the biomechanical, as well as the neuromuscular condition of the individual. The aim of this work was to examine the ability of the method to detect, through the observed changes in the shape and/or the size of the calculated basins of attraction, (a) the inherent differences between different systems (in the current application, postural restoring systems of different individuals) and (b) induced changes in the same system (the postural restoring system of an individual). The results of the study confirm the validity of the method and furthermore justify its robustness.
\end{abstract}

(c) 2013 Elsevier Inc. All rights reserved.

\section{Introduction}

\subsection{Obtaining the basin of attraction of a dynamical system}

The problem of estimating the geometry of the basin of attraction of a dynamical system from experimental data is fundamental and challenging both from the theoretical and the applied point of view.

Analytical methods can be found in the literature, obviously limited to dynamical systems described by known, analytically manageable functional forms [1-10]. Numerical procedures have also been proposed for the calculation of the basin boundaries from first principles [9,11,12]. Methods such as the so-called cell mapping method [13] have been used. There has also been work on estimating the basin of attraction from experimental data [14-16] however in general such studies are concerned with the determination of the basin's lower/upper limits or its boundaries [17].

An interesting method for numerically determining the basin of attraction from experimental data can be found in [2]. Using as application a driven two-well magneto-mechanical oscillator, the basins of attractions for the system are obtained by an ensemble of initial conditions generated by switching between stochastic and deterministic excitation.

In our previous work [18], we presented a method of numerically estimating the shape and the size of the basin of attraction from the density of the experimental data. This method uses numerical optimization and data modeling tools to obtain

\footnotetext{
* Corresponding author.

E-mail address: marzak@science.tuc.gr (M.S. Zakynthinaki).
} 
analytical curves that describe both the contours and the boundary of the basin. Finally a three-dimensional function describing the basin of attraction is calculated.

The present work presents.

- a modification of the previous method [18], providing the three-dimensional basin of attraction in the form of a threedimensional potential well and

- results of the application of the modified method to the study of the detection of changes in the geometry of the basin of attraction.

\subsection{The application: the dynamic process of balance restoration after perturbation from the vertical}

Given a sufficiently small perturbation, the human body has the ability to regain balance, following a complicated path back to the position of quiet vertical stance $[19,20]$. For vertical balance to be maintained, a variety of possible dynamics across different joints and body parts is recruited and a number of motor and sensory pathways contribute to its control [21]. Quiet stance and the maintenance of vertical balance is therefore an important motor function in humans.

The postural restoring response is a function of body morphology, muscular strength, and neurological condition; it can be affected by a number of factors, such as for example age, specific training, medication, impaired cognition, visual impairment, or even nutritional deficiencies. Injuries can also importantly affect balance, as the movement patterns leading to balance are changed so as to protect the injured area [22].

Quiet stance is rather a dynamic than a static phenomenon [23,24], as balance in quiet vertical stance does not imply motionless stability. The body's center of gravity oscillates spontaneously and continuously at low amplitude, a phenomenon known as postural sway [19,25,26]. Postural sway, still a matter of scientific discussion, is indistinguishable from correlated noise [27]. It has been suggested [23] that it is the body's clever strategy to maintain balance in vertical stance, instead of the result of an "imperfectness" of the biological system.

Although there is a lot of discussion, the integration of several mechanisms have been proposed, examples being vestibular sensing of head movement and gravitational orientation, visual sensing of self-movement, and proprioceptive sensing of ankle movement $[21,28]$. Two distinct re-stabilization mechanisms have been introduced, together with their combination: the "ankle strategy" and the "hip strategy" [23,24,29-33]. It seems that the postural system reduces the unknown, high number of the degrees of freedom of the stabilizing mechanisms by compressing them into these two muscular synergies at the neuromuscular level [24,32]. Static stability regions with respect to ankle and hip joints have been proposed [34], while the authors in [35] used a concept based on the mechanical limit of vertical stance with respect to the angles of the ankle and the hip, called stability cone [18].

Tools from non linear dynamics have been found to be very useful in understanding the complex behavior of vertical balance $[18-20,22,24,25,36]$. It has been furthermore shown that the relative motion of the ankles and the hips in postural oscillations exhibit typical hallmarks of dynamical systems, including relaxation time after perturbation [24,37]. From the point of view of dynamical systems, the difficult task of understanding the complex mechanism of the recuperation and maintenance of vertical balance can be facilitated by reducing the system's dimensionality $[18,24,25]$.

The present study is based on a dynamical systems model $[18,22,24,36]$ that does not make use of the individual components of the human body, or the non-linear interactions between them. Instead, it considers the process of dynamically regaining balance as movement inside a basin of attraction $[18,22,24]$ that corresponds to the set of the correctable angles that an individual can lean to and still and reverse the motion so as to regain vertical stance (prevent falling). The important question of how best to stabilize the vertical position is this way transformed into the question of how to achieve a wider basin of attraction, with a stronger attractor at vertical stance.

The method [18] numerically calculates the basin of attraction that corresponds to an individual from experimental data, with the aim to demonstrate that the biomechanical differences and acquired asymmetries in the body's alignment are reflected as differences in the shape and the size of the basin of attraction of human balance. The method used in the present study can be used as an important and fundamental tool for the detection of changes of the dynamics of the system under study $[18,22,24,36]$ and has potential applications that are not limited to the area of human balance, but can be extended to any other dynamical system where an estimation of its basin of attraction from experimental data is desired.

\section{Theory/calculation}

In what follows we provide a brief introduction to the model previously presented in [18,24], see also [22,36]:

\subsection{The model on the phase space of $\left(\theta_{x}, \theta_{y}\right)$}

Denoting as $F_{z}$ the vertical component of the resultant ground reaction force and $F_{x}, F_{y}$ its components on the $x$ (anteroposterior) and $y$ (mediolateral) directions respectively, then the model [24] assumes as only variables the two angles $\left(\theta_{x}, \theta_{y}\right) \in\left[-\frac{\pi}{2}, \frac{\pi}{2}\right]$, where $\theta_{x} \equiv \tan ^{-1}\left(F_{x} / F_{z}\right)$ and $\theta_{y} \equiv \tan ^{-1}\left(F_{y} / F_{z}\right)$. 


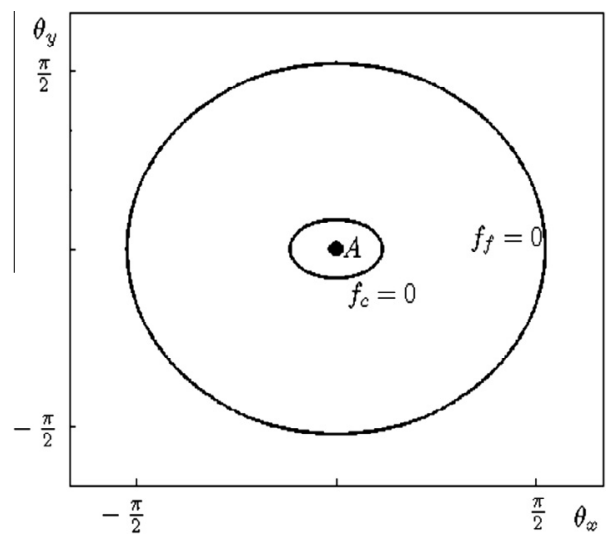

Fig. 1. The features of the model on the phase space of $\left(\theta_{x}, \theta_{y}\right)$. The attracting region $A$ around $(0,0)$ corresponds to vertical stance, the attracting circle $f_{f}\left(\theta_{x}, \theta_{y}\right)$ models failure to regain vertical balance and the critical curve $f_{c}\left(\theta_{x}, \theta_{y}\right)$ separates their basins of attraction. This figure shows an idealized critical curve.

The phase space of $\left(\theta_{x}, \theta_{y}\right)$ includes a region where postural balance can be maintained. On this region vertical stance can be represented [24] as an attracting fixed point at $\left(\theta_{x}=0, \theta_{y}=0\right)$, see Fig. 1. The set of sufficiently small "critical" angles $\theta_{x}^{c}$ and $\theta_{y}^{c}$ such that, for $0 \leqslant \theta_{x} \leqslant \theta_{x}^{c}$ and $0 \leqslant \theta_{y} \leqslant \theta_{y}^{c}$ vertical stance can be regained after an initial perturbation away from the vertical, constitutes the "critical curve", which has been modeled as a repelling set of fixed points. Beyond a certain level of initial perturbation, humans are "attracted" to falling: the subject will abandon the specified foot position resulting in actions such as falling or taking a step to prevent falling. On the phase space of $\left(\theta_{x}, \theta_{y}\right)$ this condition is represented as an attracting circle $\theta_{x}^{2}+\theta_{y}^{2}=(\pi / 2)^{2}$, see Fig. 1 . The critical curve can be viewed as the boundary between the two basins of attraction, that contain either vertical stance or horizontal failure $[18,22,24,36]$. Any muscle imbalances or asymmetries often cause deformations in the size or the shape $[22,36]$ of this critical curve.

The dynamical system [24] of dynamic upright posture restoration described on the phase space of $\left(\theta_{x}, \theta_{y}\right)$ is governed by the following set of coupled ordinary differential equations:

$$
\begin{aligned}
& \dot{\theta}_{x}=-f_{\alpha x}\left(\theta_{x}, \theta_{y}\right) f_{c}\left(\theta_{x}, \theta_{y}\right) f_{f}\left(\theta_{x}, \theta_{y}\right), \\
& \dot{\theta}_{y}=-f_{\alpha y}\left(\theta_{x}, \theta_{y}\right) f_{c}\left(\theta_{x}, \theta_{y}\right) f_{f}\left(\theta_{x}, \theta_{y}\right),
\end{aligned}
$$

where the functions $f_{\alpha x}\left(\theta_{x}, \theta_{y}\right)$ and $f_{\alpha y}\left(\theta_{x}, \theta_{y}\right)$ control the attractor at vertical state,

$$
f_{a x}\left(\theta_{x}, \theta_{y}\right)=-\left(\alpha \theta_{x}+\eta \theta_{y}\right), \quad f_{a y}\left(\theta_{x}, \theta_{y}\right)=-\left(\gamma \theta_{x}+\kappa \theta_{y}\right)
$$

the function $f_{c}\left(\theta_{x}, \theta_{y}\right)$ models the repelling critical curve (see below) and the function $f_{f}\left(\theta_{x}, \theta_{y}\right)$ models the attracting circle of failure to regain vertical stance,

$$
f_{f}\left(\theta_{x}, \theta_{y}\right)=\frac{\pi^{2}}{4}-\theta_{x}^{2}-\theta_{y}^{2}
$$

It should be noted here that a linear stability analysis of the model regarding movement on the phase space of $\left(\theta_{x}, \theta_{y}\right)$ has been performed [24], and a modification of the model has been presented and analyzed [22] to account for rotated and/ or skewed movement patterns. Furthermore, a detailed method of the derivation of the critical curve $f_{c}\left(\theta_{x}, \theta_{y}\right)$ from experimental data has been presented, see [36].

The present study goes one step forward by taking into account the existence of body sway at quiet vertical stance. This way the attractor at $(0,0)$ is not considered here as a point but rather as a small attracting region around the vertical [18]. Assuming that this attracting region is an ellipse of major semi-axis $R_{1}$ and minor semi-axis $R_{2}$ then all the points that lie inside this elliptical region are considered to belong to the attractor of vertical stance. From the biomechanical point of view, the larger the size of the ellipse, the stronger the person's ability to maintain balance.

\subsection{The basin of attraction of human balance}

Following the model presented in [18], the motion of the center of gravity of the body is considered to take place inside a two-dimensional manifold (a basin of attraction), the shape and size of which is characteristic of the movement patterns and asymmetries of the individual and is independent of the initial perturbation from quiet stance [18]. A mentioned above, this basin of attraction includes the attractor at vertical stance and its boundary is the critical curve which separates the condition of being able to regain balance with the condition of the horizontal failure state. It is only inside this basin of attraction that initial perturbations from the vertical can be corrected, with the orbit spiraling back onto the attractor of vertical stance. 
In the sections that follow the phase space of the two angles is expressed as a polar coordinate system $(\rho, \theta)$ (see also [18]) instead of the cartesian $\left(\theta_{x}, \theta_{y}\right)$, such that

$$
\rho \equiv \sqrt{\theta_{x}^{2}+\theta_{y}^{2}}
$$

$\rho \geqslant 0$ and

$$
\theta \equiv \arctan \left(\theta_{y} / \theta_{x}\right)
$$

$\theta=0, \ldots, 2 \pi$.

To model the contours that cut the basin of attraction at different depths $z$ we consider that each contour is represented by a closed curve on the $(\rho, \theta)$ plane. For a three-dimensional representation of the basin of attraction of the system of human balance, let us denote as $z(\rho, \theta)$ the local depth of the basin at $(\rho, \theta)$. In order to estimate the basin of attraction, $z(\rho, \theta)$ is derived from experimental data, following the method described in [18]: given an integer $N$ that defines the resolution of the basin of attraction, the phase space $(\rho, \theta)$ of the system is partitioned into an $N \times N$ grid and the number of experimentally recorded data points that are contained within each of the $N^{2}$ boxes are counted to provide the density distribution $\mathfrak{D}(\rho, \theta)$ of the experimental data. On the boundary of the basin of attraction (i.e. on the critical curve) there is $\mathfrak{D}=0$ and, as the attractor of vertical stance within the basin is approached, the values of $\mathfrak{D}$ become very large. There is always $\mathfrak{D} \geqslant 0$.

The depth $z$ can be obtained from the values of the density distribution $\mathfrak{D}$, this way reflecting the number of experimentally recorded points at a given $(\rho, \theta)$. In other words, the longer the system resides in a neighborhood of the basin of attraction, the deeper the basin there [18]. With the aim to derive a basin of attraction that will be a realistic approximation of a potential well and this way applicable for simulations the method presented in [36] has been modified. The depth $z$ of the basin of attraction is derived as follows:

$$
z(\rho, \theta) \equiv \frac{1}{\mathfrak{D}(\rho, \theta)+1},
$$

this way assuming that:

- the plane $z=1$ corresponds to the critical curve (the boundary of the basin of attraction),

- as we move further inside the basin approaching the attractor of vertical stance, the values of $z$ decrease,

- the region $z \ll 1$ (practically the plane $z=0$ ) corresponds to the attracting region of vertical stance at the heart of the basin of attraction, and

- inside the basin there is $0 \leqslant z \leqslant 1$.

In order now to obtain the three-dimensional function describing the basin of attraction, the functions that give the contours of the basin are first calculated as follows (see also [18]): Given an integer $m \in \mathbb{N}$, an appropriate algorithm [18] is applied to find the region on the phase space of $(\rho, \theta)$ inside which $\mathfrak{D}(\rho, \theta) \geqslant m$. Then the contour that corresponds to

$$
z=\frac{1}{m+1}, \quad m \in \mathbb{N}
$$

is calculated as the closed curve enclosing this region, by means of numerical curve fitting algorithms, see also [18]. The contour corresponding to $z=1$ is the critical curve.

In the present study the Levenberg-Marquardt algorithm (LMA) [38], also known as the damped least-squares (DLS) method, is used for the numerical curve fitting purposes. The LMA interpolates between the Gauss-Newton and the gradient descent methods, and is a very popular curve-fitting algorithm used in many numerical applications.

We assumed the functions describing the contours of the basin of attraction to be sums of ellipses on the phase space of $(\rho, \theta)$. This way the contour that corresponds to a given depth $z$ has the form

$$
\rho_{z}(\theta)=\sum_{i=1}^{M z} \rho_{z, i}(\theta),
$$

where $M z \in \mathbb{N}^{t}$ is the number of ellipses that sum up to construct the contour that corresponds to each $z$. The components $\rho_{z, i}(\theta)$ are ellipses of the general polar form

$$
\rho_{z, i}(\theta)=\frac{P_{z, i}(\theta)+Q_{z, i}(\theta)}{R_{z, i}(\theta)},
$$

where

$$
\begin{aligned}
& P_{z, i}(\theta)=r_{0 z, i}\left[\left(b_{z, i}^{2}-a_{z, i}^{2}\right) \cos \left(\theta+\theta_{0_{z, i}}-2 \phi_{z, i}\right)+\left(a_{z, i}^{2}+b_{z, i}^{2}\right) \cos \left(\theta-\theta_{0 z, i}\right)\right], \\
& Q_{z, i}(\theta)=\sqrt{2} a_{z, i} b_{z, i} \sqrt{R_{z, i}(\theta)-2 r_{0_{z, i}}^{2} \sin ^{2}\left(\theta-\theta_{0 z, i}\right)}, \\
& R_{z, i}(\theta)=\left(b_{z, i}^{2}-a_{z, i}^{2}\right) \cos \left(2 \theta-2 \phi_{z, i}\right)+a_{z, i}^{2}+b_{z, i}^{2}
\end{aligned}
$$


and $a_{z, i}, b_{z, i}, r_{0 z i, i}, \theta_{0 z, i}$ and $\phi_{z, i}$, are constants that are appropriately chosen for a given $z$.

The choice of the above function combinations was based on the fact that the experimental error can unavoidably affect the fine structure of the contours and as a result of the three-dimensional basin of attraction. For this reason no emphasis was given on calculating the details of the contours' shape. The functions $\rho_{z}(\theta)$ that describe each contour are obtained through numerical optimization $[18,36,38]$, so as to provide best fit to the experimental data.

On the three-dimensional (cylindrical) space of $(z, \rho, \theta)$ the basin of attraction has the shape of a well and is assumed to be obtained by rotating a gaussian bell around the $z$ axis and vertically to the phase space $(\rho, \theta)$ of the system. The shape and the position of the gaussian bell depends on $\theta$ as follows:

- its mean $\mu(\theta)$ follows the critical curve, i. e. $\mu(\theta)=\rho_{1}(\theta)$

- its standard deviation $\sigma(\theta)$ is obtained by use of the contour at $z_{\sigma}=e^{-1}$, i.e. $\sigma(\theta)=\mu(\theta)-\rho_{z_{\sigma}}(\theta)$.

The three dimensional function that describes the basin of attraction (the potential function) is therefore expressed in the form:

$$
z(\rho, \theta)=\exp \left\{-\left[\frac{\rho(\theta)-\rho_{1}(\theta)}{\rho_{1}(\theta)-\rho_{z_{\sigma}}(\theta)}\right]^{2}\right\} .
$$

It should be noted here that, as the value of $z_{\sigma}$ does not correspond to an integer value of $m$, see Eq. (1), the contour $\rho_{z_{\sigma}}(\theta)$ could not be obtained directly from the experimental data. For this reason it was numerically approximated to be $\rho_{z_{\sigma}}(\theta) \approx 1.103 \rho_{z_{2}}(\theta)$, where the depth $z_{2}$ corresponds to $m=2$, see Eq. (1).

\section{Material and methods}

\subsection{The subjects of the study}

Three subjects served as test subjects for the application presented in this study:

1. Subject "A": A healthy 40 year old male ex-high level track-and-field athlete who was still keeping physically active. Two sets of experimental data were collected in two different data collection sessions. Care was taken to minimize the time between the two successive data collection sessions so as to avoid any possible effect of training and/or change in the alignment of the body and therefore the movement patterns of the subject.

2. Subject "B": A healthy 49 year old male professional Judoka (5th Dan).

As with the first subject, two sets of experimental data were collected in two different occasions, while care was taken so that the time interval between the two data collection sessions did not allow for any modifications in the subject's movement patterns.

3. Subject "C": A 21 year-old male elite athlete of the Spanish national Judo team, who had suffered a rupture of the anterior cruciate ligament $(\mathrm{ACL})$ and the lateral meniscus of the right knee. This type of injury is in general very common and in Judo is one of the most common injuries, usually occurring during competition with a probability of approximately $30 \%$ [39]. The injury was recovered by surgical reconstruction of the ligament and a rehabilitation program of two sessions a day five days a week of duration of $1.5 \mathrm{~h}$ each and for a total period of five and a half months. The treatment consisted of electrotherapy and ultrasound, as well as proprioception exercises and strengthening of flexo-extensor muscles of the knee, cyriax massage and exercises in aquatic environment. The main objective of an athlete who has suffered such an injury and has undergone through a ligament reconstruction surgery is to restore the stability of the knee so that they can return to the same level of physical activity as soon as possible [40].

As the aim of this study was to detect the changes in the Judoka's balance patterns resulting from the rehabilitation after the injury, the basin of attraction of this subject was calculated using two different data sets recorded:

(a) while the subject was injured, a week before the surgical intervention

(b) a few months after the end of the rehabilitation period, when the athlete had fully recovered from his injury.

The authors confirm that our research met the highest ethical standards for authors and co-authors and that it was performed following the guidelines of the Declaration of Helsinki, last modified in 2008.

In addition, the authors certify that the present research was carried out in the absence of any financial, personal or other relationships with other people or organizations that could inappropriately influence, or be perceived to influence, the presented work and lead to a potential conflict of interest.

\subsection{The protocol}

The experimental data collection required a force platform, able to record the time series of the ground reaction force that the subjects exerted while trying to regaining balance after an initial voluntary perturbation. A Kistler 9286AA portable force 
platform and its corresponding software was used, with a sampling rate of $200 \mathrm{~Hz}$ and a total recording time of $5 \mathrm{~s}$ for each complete movement. The original recorded data did not undergo any filtering or editing before analysis.

The present study followed the same experimental protocol described in $[22,18,36]$. The subjects initially stood on the force platform with hands on hips, eyes open, and focused on a spot on the wall. Care was always taken so as to assure that the experiment was carried out in a quiet room (a laboratory) free from distractions. During experimental data collection the subjects were asked to repeat maximum amplitude and speed voluntary movements in the directions forward, backward, left, right, and the four diagonals forward-left, forward-right, backward-left and backward-right. The time series of the ground reaction force were recorded for five successful movement for each of the eight directions (giving a total of 40 time series for each data set). A successful movement consisted of the subject being able to correct the initial perturbation and regain vertical stance. The subjects were allowed to bend at ankles, knees, or waist or twist their body in order to correct the perturbation, as long as their hands remained on their hips and their feet on the floor.

\subsection{The phase space of the experimentally recorded data}

From the three components $F_{x}, F_{y}$ and $F_{z}$ of the ground reaction force recorded by the force platform, the angles $\theta_{x}$ (between $F_{z}$ and $F_{x}$ ) and $\theta_{y}$ (between $F_{z}$ and $F_{y}$ ) were obtained. Figs. 2-4 present the experimentally recorded sets of 40 time series data, plotted on the phase space of $\left(\theta_{x}, \theta_{y}\right)$. For the data shown in these figures, positive values of $\theta_{x}$ point forwards and positive values of $\theta_{y}$ point to the left.

By mere observation of these figures, it can be easily seen that the first two subjects do not show any significant changes in their movement patterns, while for the third subject the changes are dramatic, implying that the rehabilitation reached

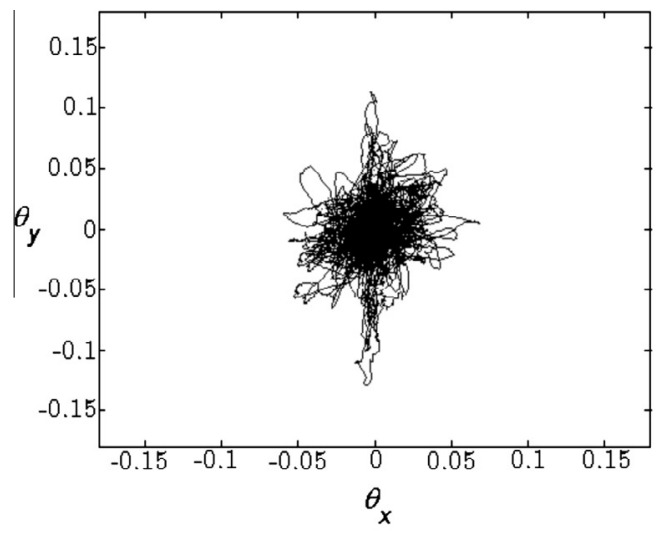

(a)

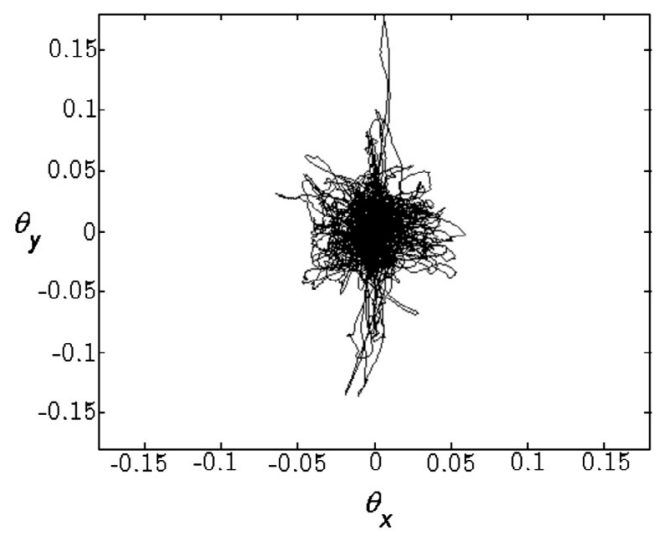

(b)

Fig. 2. Subject "A": The experimentally recorded time series data converted into angles and plotted on the phase space of $\left(\theta_{x}, \theta_{y}\right)$. (a): First data collection session, (b): second data collection session. The angles are expressed in radians.

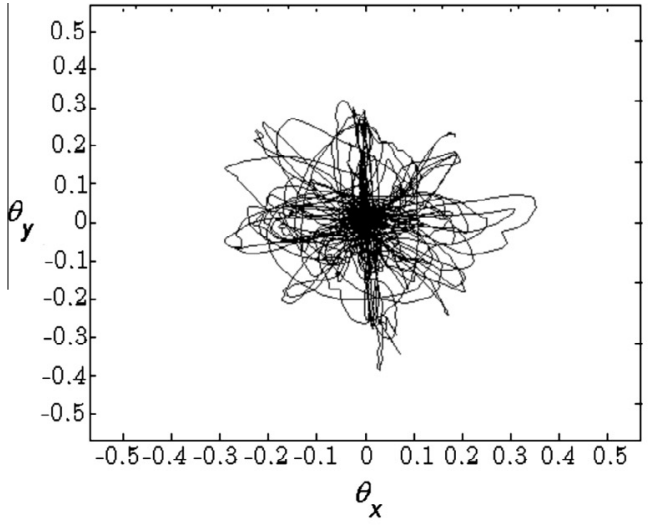

(a)

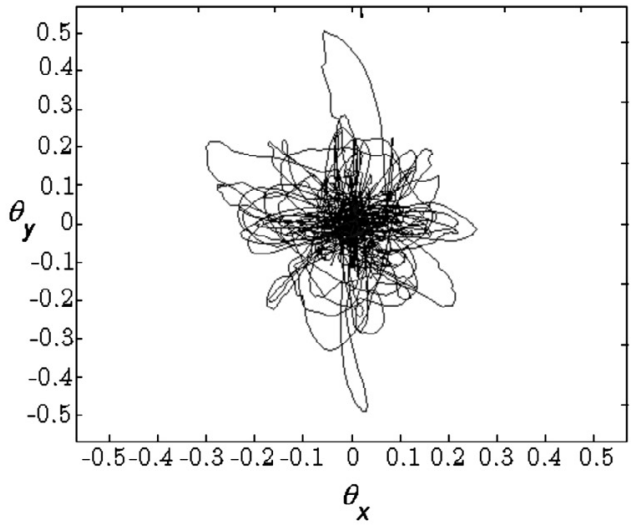

(b)

Fig. 3. Subject "B": The experimentally recorded time series data converted into angles and plotted on the phase space of $\left(\theta_{x}, \theta_{y}\right)$. (a): First data collection session, (b): second data collection session. The angles are expressed in radians. 


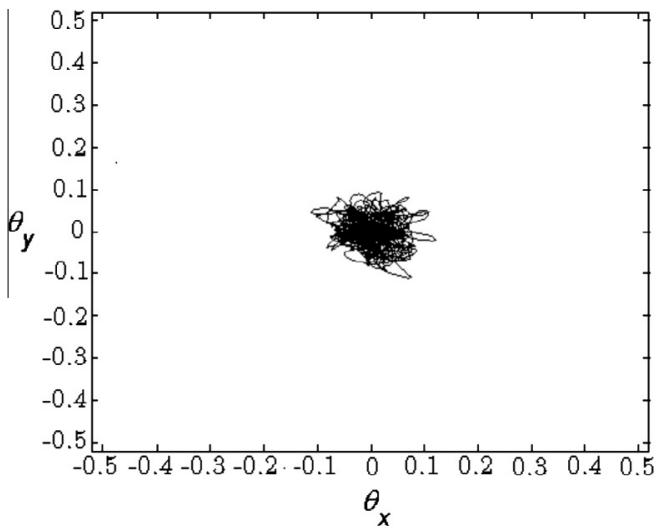

(a)

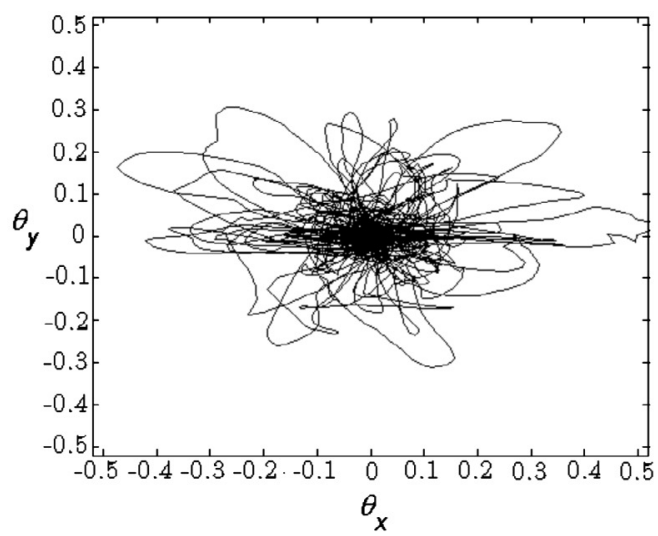

(b)

Fig. 4. Subject "C": The experimentally recorded time series data converted into angles and plotted on the phase space of $\left(\theta_{x}, \theta_{y}\right)$. (a): First data collection session (injury), (b): second data collection session (after rehabilitation and recovery). The angles are expressed in radians.

exceptional levels. Regarding subject " $C$ ", the effects of the injury are clearly shown, not only as restrictions in the range of movement, but also as limited movement on the $\left\{\theta_{x}<0, \theta_{y}<0\right\}$ part of the phase space. The differences in the range of the maximum angular movements between the three subjects should be also emphasized (see also Section 4.2 below).

\section{Results}

\subsection{The attracting regions at vertical stance}

As explained in Section 2.1, in order to take into account the presence of body sway, the attractor of vertical stance within the basin is not modeled as a point but rather as a small attracting region around the vertical.

These attracting regions were calculated from the experimental data to be:

- for subject "A",

$\rho_{0}=0.027$ (circular region),

- for subject "B", $\rho_{0}=0.012$ (circular region),

- for the pre-operation condition of subject "C",

$$
\rho_{0}=\frac{0.0000945 \sqrt{2}}{\sqrt{0.00002925 \sin (2 \theta)+0.00019125}}
$$

(a small elliptical region with principal axis $a_{0}=0.0105$, secondary axis $b_{0}=0.009$, rotated by $\phi_{0}=-\pi / 4$ ),

- for the post-operation and rehabilitation condition of subject " $\mathrm{C}$ ",

$$
\rho_{0}=0.013 \text { (circular region). }
$$

According to the experimental values above, for all three healthy and active subjects that participated in this study the small attracting region around the vertical can be modeled as a circle of radius $R$ (the values of $R$ in the relations above are expressed in radians), with the pre-operation condition of subject " $C$ " being the only exception, as the symmetry of the attractor at vertical stance was affected by the injury.

\subsection{The range of movement}

Denoting as $\theta_{\max }$ the maximum (in absolute terms) recorded angle that a subject could lean to and still regain vertical stance, then the value of $\theta_{\max }$ gives a good estimation of the subject's range of movement and subsequently of their muscular strength, flexibility and general ability to regain balance, see also [18,22,24,36,41].

The analysis of the present study revealed angular limits of the basins for each subject (see also Figs. 10-13):

- for subject " $A$ ", there is $\theta_{\max A}=0.17$ radians.

- for subject " $\mathrm{B}$ ", there is $\theta_{\max B}=0.5$ radians.

- for the pre-operation condition of subject " $\mathrm{C}$ ", there is $\theta_{\operatorname{maxC1}}=0.115$ radians.

- for the post-operation and rehabilitation condition of subject " $\mathrm{C}$ ", there is $\theta_{\max C 2}=0.51$ radians. 


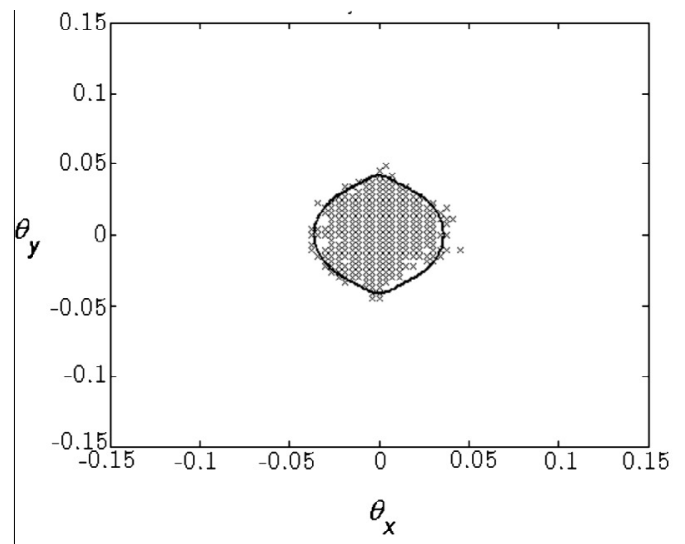

(a)

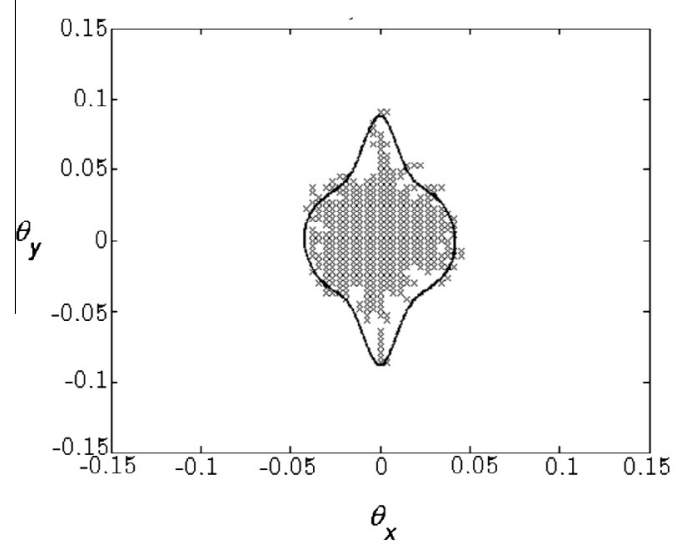

(c)

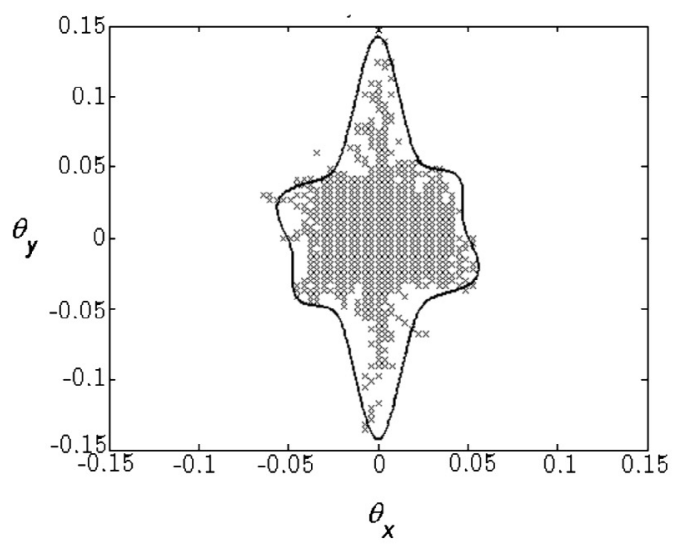

(e)

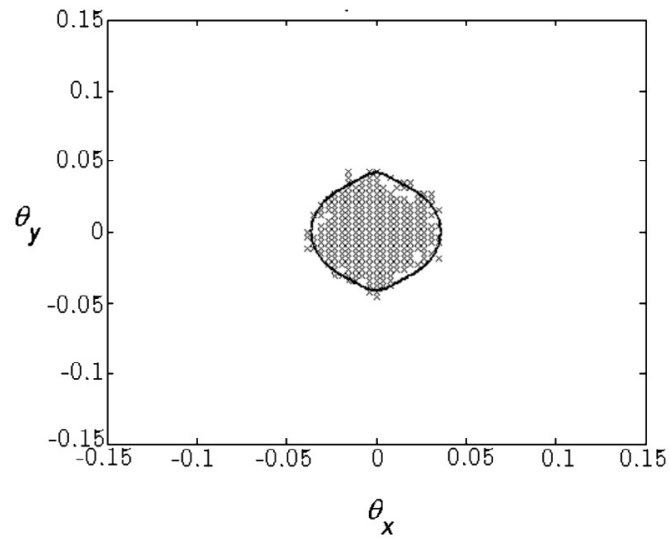

(b)

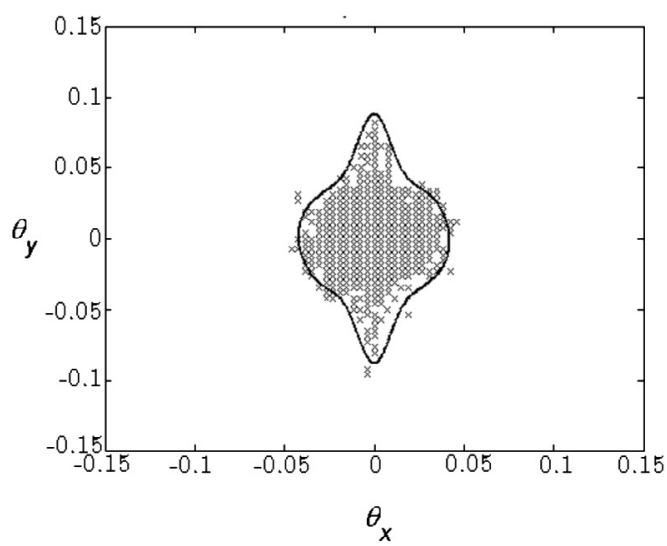

(d)

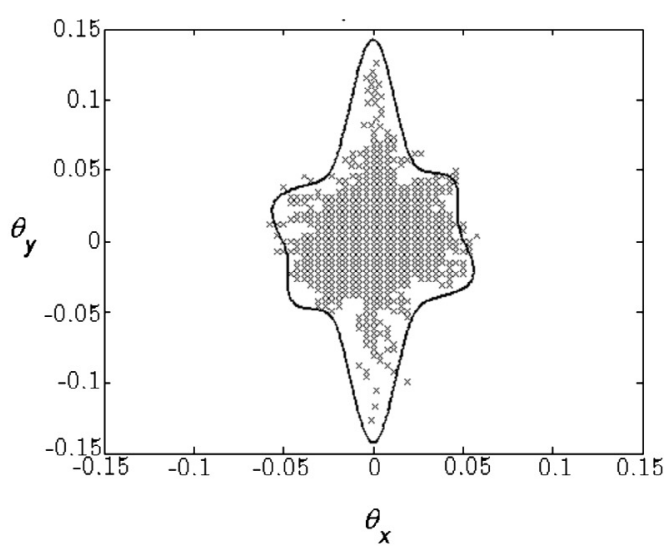

(f)

Fig. 5. Grey points: experimental data points of subject " $A$ ", on the phase space of $\left(\theta_{x}, \theta_{y}\right)$. Solid curves: calculated contours enclosing regions of less or equal depth. First data collection session: (a) $z=0.09$, (c) $z=0.2$, (e) $z=0.5$; second data collection session: (b) $z=0.09$, (d) $z=0.2$, (f) $z=0.5$. The angles are expressed in radians.

\subsection{The contours of the basins of attraction}

As mentioned in the previous sections, the contours of the basin of attraction, each of which corresponds to a constant depth $z$, are calculated by use of appropriate curve fitting, see also [18,38]. Fig. 5 presents examples of the calculated contour curves for the data belonging to the first subject, together with the data points (grey points). Three different contours are 
presented, for $z=0.09, z=0.2$ and $z=0.5$ that are calculated by use of the same function for a particular $z$, for both data collection sessions.

Fig. 5 also illustrates and emphasizes the robustness of the method and its lack of sensitivity to different experimental data sets (as long as these data sets correspond of course to the same, unmodified, condition of a subject). Even though occasional differences in the data points can be observed, they are of minor importance: within the range of experimental error, the data sets shown in 5 can be assumed to be indistinguishable and independent of the data collection session.

Regarding now maximum movements, the differences in the recorded data between the two data collection sessions seem to be more significant (please refer to the full data sets, shown in Figs. 2 and 3). Such deviations are related to differences in the subjects' motivation or their general biomechanical or neuromuscular condition during the data collection sessions and are to be expected, even from carefully chosen subjects such as those that took part in the present study. For this reason, the critical curve (contour $z=1$ ) and subsequently the border of the basin of attraction was calculated as the curve enclosing the superposition of the two data sets (of the first and the second data collection session), so that all maximum possible movements were taken into account.

Hence, while the contours of the interior of the basins of attraction can be precisely calculated, the boundaries of the basin of attraction, i.e. the critical curves, can only be estimated. This limitation arises from the fact that the method is based on experimental data of human subjects, so absolute maximum movements are, by definition, almost impossible to be recorded. However, even though the differences in the maximum recorded movements between the two data collection sessions can not be totally avoided, they can be minimized: an effective approach to achieve minimum differences is to use highly trained subjects, able to control their body movements, such as the athletes used in this study.

Fig. 6 shows the set of the contours that was used for the calculation of the basin of attraction of subject "A". The same procedure was followed in order to obtain the contours of the basins of attraction that correspond to subject "B", as well as the two (pre-and post-operation) conditions of subject "C", see Figs. 7-9,. Appendix A includes, as an example, the numerically fitted functions that describe the contours of the basin of attraction of subject "B".

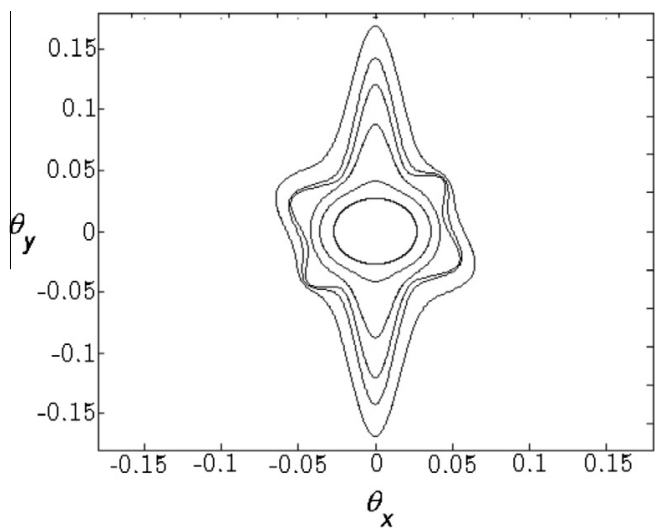

Fig. 6. The set of calculated contours of the basin of attraction of Subject "A", corresponding (from the smallest to the largest) to $z=0.04, z=0.09, z=0.2, z=0.33, z=0.5$ and $z=1$. The angles are expressed in radians.

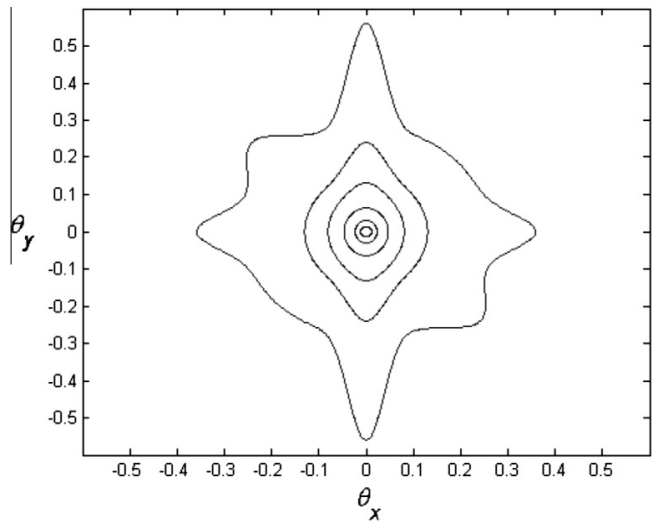

Fig. 7. The set of calculated contours of the basin of attraction of Subject "B", corresponding to $z=0.04, z=0.09, z=0.2, z=0.33, z=0.5$ and $z=1$. The functions describing these contours are indicatively given in Appendix A. The angles are expressed in radians. 


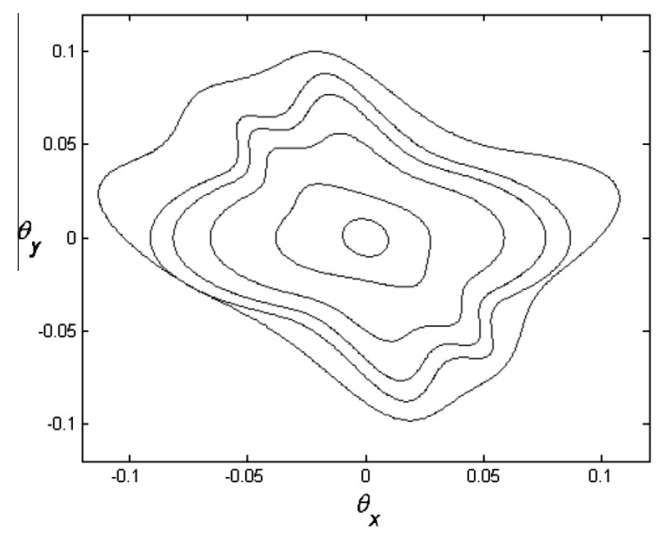

Fig. 8. The set of calculated contours of the basin of attraction of the pre-operation condition of Subject " $C$ ", corresponding to $z=0.04, z=0.09, z=0.2, z=0.33, z=0.5$ and $z=1$. The angles are expressed in radians.

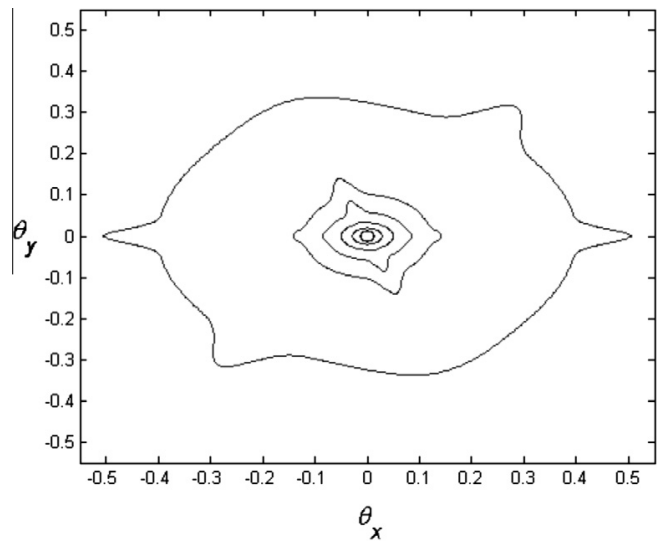

Fig. 9. The set of calculated contours of the basin of attraction of the post-operation condition of Subject " $C$ ", corresponding to $z=0.04, z=0.09, z=0.2, z=0.33, z=0.5$ and $z=1$. The angles are expressed in radians.

\subsection{The three dimensional basins of attraction}

Figs. 10-13 show the three-dimensional plots of the interior of the basins of attraction, see Eq. (3), for subject " $A$ ", subject " $\mathrm{B}$ " and the two different conditions of subject "C" respectively.

\section{Discussion}

The present application of the method has the following potential biomechanical conclusions:

- Subject "A":

The basin of attraction that corresponds to subject " $A$ " has a strong base, the larger attracting region around the vertical of all three subjects, reflecting a better ability to maintain balance at vertical stance. His overall range of movement is, though, far more restricted, in comparison to the other two subjects. The walls of the basin are steep, meaning that every perturbation away from the vertical, small enough so that it does not exceed the small limits of the basin, is rapidly corrected.

The biomechanics of subject " $A$ " seems very conservative, as it does not allow his center of mass to take risks away from the vertical. Taking into account that subject " $A$ " is a track-and-field athlete, this could be due to the combination of strong leg muscles and a rather limited flexibility.

- Subject "B":

The general condition of subject " $\mathrm{B}$ " could be classified excellent; the range of movement is wide and the corresponding basin of attraction is symmetric enough with walls that are far less abrupt that those of subject " $A$ ".

Subject "B" is a high level Judoka (as well as subject "C"), a fact that fundamentally implies a powerful combination of muscular strength and flexibility. 


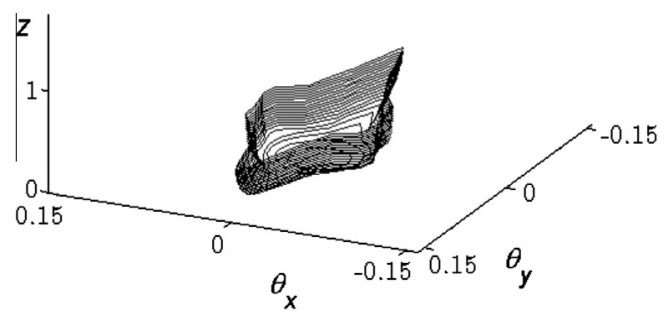

Fig. 10. Subject "A", $z(\rho, \theta)$ : three-dimensional plot of the interior of the calculated basin of attraction, $0 \leqslant z \leqslant 1,0 \leqslant \rho \leqslant \rho_{1}(\theta)$. The angles are expressed in radians.

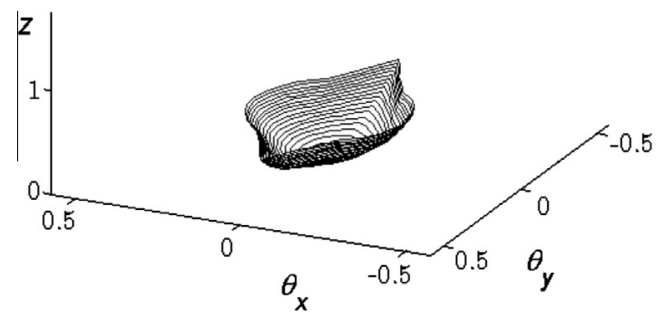

Fig. 11. Subject "B", $z(\rho, \theta)$ : three-dimensional plot of the interior of the calculated basin of attraction, $0 \leqslant z \leqslant 1,0 \leqslant \rho \leqslant \rho_{1}(\theta)$.The angles are expressed in radians.

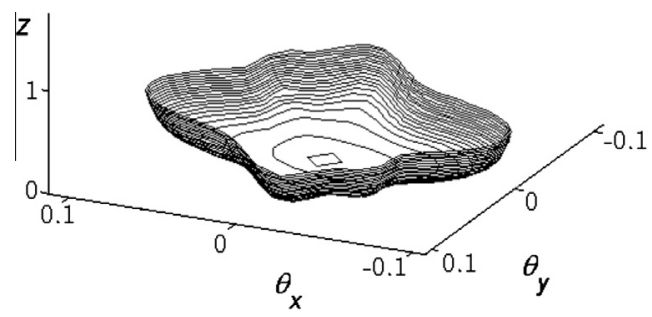

Fig. 12. Subject " $C$ ", pre-operation condition, $z(\rho, \theta)$ : three-dimensional plot of the interior of the calculated basin of attraction, $0 \leqslant z \leqslant 1,0 \leqslant \rho \leqslant \rho_{1}(\theta)$. The angles are expressed in radians.

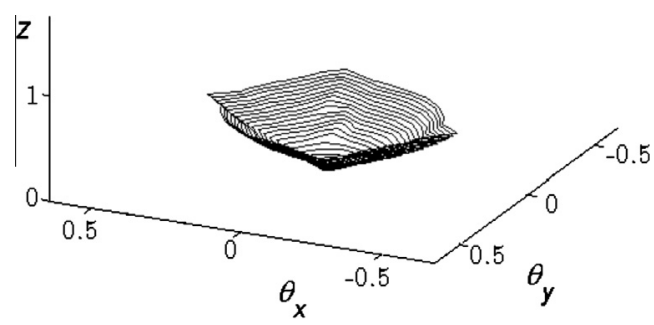

Fig. 13. Subject " $\mathrm{C}$ ", post-operation condition, $z(\rho, \theta)$ : three-dimensional plot of the interior of the calculated basin of attraction, $0 \leqslant z \leqslant 1,0 \leqslant \rho \leqslant \rho_{1}(\theta)$. The angles are expressed in radians.

\section{- Subject "C":}

The effects of the injury of subject " $\mathrm{C}$ " are clearly reflected on the size, as well as the shape of the attracting region at vertical stance. There is a general absence of movement on the $\left\{\theta_{x}<0, \theta_{y}<0\right\}$ part of the phase space of the system's variables, apparently adopted by the subject's biomechanics in order to protect the injured knee. The basin of attraction that corresponds to the injured period of subject " $\mathrm{C}$ " carefully imposes strong restrictions on the subject's range of movement; vertical stance of the injured subject is easily lost.

After recuperation from the injury, the shape and the size of the central parts of the basin are corrected and slightly expanded, while an exceptional opening of the walls has been accomplished. To illustrate this more clearly, Fig. 14 shows the two basins of attraction that correspond to the pre- and post-operation conditions of subject " $\mathrm{C}$ ", on the same plot. Such changes are obviously the achievement of an extraordinary treatment and rehabilitation program. 


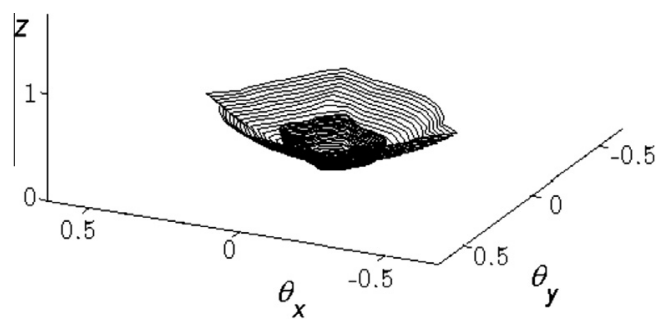

Fig. 14. Subject " $C$ ", plots of the interior of $z(\rho, \theta)$ of the pre- and post-operation conditions shown on the same coordinate system. For each of the plots there is $0 \leqslant z \leqslant 1,0 \leqslant \rho \leqslant \rho_{1}(\theta)$. The angles are expressed in radians.

\section{Conclusions}

In this work we applied a previously presented model [18] that considers the process of regaining vertical stance as movement inside a basin of attraction. This basin of attraction can be considered as a potential well, inside which the center of mass moves during the recuperation of vertical stance after the initial perturbation.

In order to assure high motivation from the subjects during data collection, as well high data quality, three high level active athletes were chosen as subjects of the study.

To conclude, we highlight the power of the presented method as well as its robustness. The validity of the method, as well as of the protocol used is confirmed by the fact that the method is able to successfully detect:

1. the inherent differences in the patterns of movement between the subjects that are a result of the differences in their training and the nature of their physical activity,

2. the impressive differences in the shape and the size of the basin of attraction achieved by the rehabilitation program and the recovery of the injury of the third subject.

The method presented here be can successfully applied not only to problems of human balance such the one presented here but also to any other problem for which the use of experimental data is necessary for the calculation of a potential well.

\section{Acknowledgement}

This work is dedicated to the memory of our collaborator, partner and friend, James Robert Stirling.

Appendix A. The numerically obtained functions that describe the contours of the basin of attraction. Example: subject “B”.

$$
\begin{aligned}
& \rho_{0}(\theta)=0.012, \\
& \rho_{0.09}(\theta)=\frac{0.00068904 \sqrt{2}}{\sqrt{0.00034385 \cos (2 \theta)+0.00142033}}, \\
& \rho_{0.2}(\theta)=\frac{0.0030315 \sqrt{2}}{\sqrt{0.00195125 \cos (2 \theta)+0.00636925}}, \\
& \rho_{0.33}(\theta)=\frac{0.007650 \sqrt{2}}{\sqrt{0.004779 \cos (2 \theta)+0.016029}}+\frac{0.000165 \sqrt{2}}{\sqrt{0.00086975 \cos (2 \theta)+0.00093025}}, \\
& \rho_{0.5}(\theta)=\frac{0.015795 \sqrt{2}}{\sqrt{0.004536 \cos (2 \theta)+0.031914}}+\frac{0.001458 \sqrt{2}}{\sqrt{0.01148175 \cos (2 \theta)+0.01184625}}, \\
& \rho_{1}(\theta)=\frac{0.036 \sqrt{2}}{\sqrt{0.0076 \cos (2 \theta)+0.0724}}+\frac{0.00270 \sqrt{2}}{\sqrt{-0.017825 \cos (2 \theta)+0.018625}}+\frac{0.00512 \sqrt{2}}{\sqrt{0.102144 \cos (2 \theta)+0.102656}} \\
& +\frac{0.0143 \sqrt{2}}{\sqrt{0.011931 \sin (2 \theta)+0.012269}},
\end{aligned}
$$




\section{References}

[1] H.A. Antosiewicz, A survey of Lyapunovs second method, volume 4 of Contributions to the Theory of Nonlinear Oscillations, Princeton University Press, 1958, pp. $141-166$.

[2] J.R. Cusumano, B.W. Kimble, A stochastic interrogation method for experimental measurements of global dynamics and basin evolution: application to a two-well oscillator, Nonlinear Dyn. 8 (1995) 213-235.

[3] W. Hahn, Theory and Applications of Lyapunovs Direct Method, Prentice-Hall, Englewood Cliffs, NJ, 1963.

[4] J.R. Hewit, C. Storey, Computer application of the tracking function approach to practical stability, Electron. Lett. 2 (1966) 408-409.

[5] A.M. Letov, Stability in Nonlinear Control Systems, Princeton University Press, 1961.

[6] A. Levin, An analytical method of estimating the domain of attraction for polynomial differential equations, IEEE Trans. Autom. Control 39 (12) (1994) 2471-2475.

[7] W.O. Paradis, D.D. Perlmutter, Tracking function approach to practical stability and ultimate boundedness, AIChE J. 12 (1966) 13-136.

[8] D.D. Perlmutter, Stability of Chemical Reactors, Prentice-Hall, Englewocd Cliffs, 1972.

[9] M.T.R. Genesio, A. Vicino, On the estimation of asymptotic stability regions: state of the art and new proposals, IEEE Trans. Autom. Control AC-30 (8) (1985) 747-755.

[10] J.P.L. Salle, S. Lefschetz, Stability by Lyapunov's Direct Method, Academic, New York, 1961.

[11] E.J. Davison, K.C. Cowan, A computational method for determining the stability region of a second-order non-linear autonomous system, Int. J. Control 9 (1969) 349-357.

[12] J. Texter, Numerical algorithm for implementing zubovs consuuction in two-dimensional systems, IEEE Trans. Automat. Control AC-19 (1974) 62-63.

[13] A.L. Schwab, M. Wisse, Basin of attraction of the simplest walking model, in: Proceedings of DETC01 ASME 2001 Design Engineering Technical Conferences and Computers and Information in Engineering Conference, 2001, pp. 1-9.

[14] J.A.Y.C. Grebogi, E. Ott, Metamorphoses of basin boundaries in nonlinear dynamical systems, Phys. Rev. Lett. 56 (10) (1986) 1011-1016.

[15] A.I. Lebedev, I. Sluchinskaya, A new method for determining parameters of the potential well of off-center atoms from EXAFS data, Crystalogr. Rep. 49 (suppl. 1) (2004) 594-598.

[16] H. Urai, New method for potential well measurements using bubble runout in ion-implanted bubble devices, IEEE Trans. Magn. MAG-21 (6) (1985) 2676-2679.

[17] K. Pakdamana, C. Grotta-Ragazzoc, C.P. Maltad, O. Arinoe, J.-F. Vibertb, Effect of delay on the boundary of the basin of attraction in a system of two neurons, Neural Networks 11 (1998) 509-519.

[18] M.S. Zakynthinaki, J.R. Stirling, C.A. Cordente, A. López, M. Sillero, G. Rodríguez, J. Sampedro, Modelling the basin of attraction as a two-dimensional manifold from experimental data: applications to balance in humans, Chaos 20 (2010) 013119-1-1.

[19] J. Milton, J.L. Cabrera, T. Ohira, S. Tajima, Y. Tonosaki, C.W. Eurich, S.A. Campbell, The time-delayed inverted pendulum: implications for human balance control, Chaos 19 (2009) 026110.

[20] J.G. Milton, Introduction to focus issue: bipedal locomotion from robots to humans, Chaos 19 (2009) 026101.

[21] B.L. Luu, T.P. Huryn, H.F.M.V. der Loos, E.A. Croft, J.S. Blouin, Validation of a robotic balance system for investigations in the control of human standing balance, IEEE Trans. Neural Syst. Rehabil. Eng. 19 (4) (2011) 382-390.

[22] M.S. Zakynthinaki, J. Madera, A. López, C.A. Cordente, G. Rodríguez, M. Sillero, J. Sampedro, Rotated balance in humans due to repetitive rotational movement, Chaos 20 (2010) 013118-1-1.

[23] M. Günther, O. Müller, R. Blickhan, Watching quiet human stance to shake off its straitjacket, Arch. Appl. Mech. 81 (2011) 283-302.

[24] J.R. Stirling, M.S. Zakynthinaki, Stability and the maintenance of balance following a perturbation from quiet stance, Chaos 14 (1) (2004) $96-105$

[25] B.G. Bardy, O. Oullier, On perturbation and pattern coexistence in postural coordination dynamics, J. Motor Behav. 39 (4) (2007) 326-334.

[26] G.E. Riccio, T.A. Stoffregen, Affordances as constraints on the control of stance, Human Mov. Sci. 7 (1988) 265-300.

[27] J.J. Collins, C.J. DeLuca, Random walking during quiet standing, Phys. Rev. Lett. 73 (1994) 764-767.

[28] M.T. Blàzquez, M.A.F.A. de Saavedra, A.M. Lallena, P. Carpena, Ankle and hip postural strategies defined by joint torques, J. Comput. Appl. Math. 233 (2010) 1478-1482.

[29] N. Fujisawa, T. Masuda, Y. Inaoka, H. Fukuoka, A. Ishida, H. Minamitani, Human standing posture control system depending on adopted strategies, Med. Biol. Eng. Comput. 43 (1) (2005) 107-114.

[30] T. Kiemel, K.S. Oie, J.J. Jeka, Slow dynamics of postural sway are in the feedback loop, J. Neurophysiol. 95 (3) (2006) $1410-1418$.

[31] A.D. Kuo, An optimal control model for analyzing human postural balance, IEEE Trans. Biomed. Eng. 42 (1) (1995) $87-101$.

[32] L.M. Nashner, G. McCollum, The organization of postural movements: a formal basis and experimental synthesis, Behav. Brain Sci. 26 (1985) 135-172.

[33] C.F. Runge, C.L. Shupert, F.B. Horak, F.E. Zajac, Ankle and hip postural strategies defined by joint torques, Gait Posture 10 (2) (1999) 161-170.

[34] B.G. Bardy, L. Marin, T.A. Stoffregen, R.J. Bootsma, Postural coordination modes considered as emergent phenomena, J. Exp. Psychol. Human Percept. Perform. 25 (1999) 1284-1301.

[35] G. McCollum, T.K. Leen, Form and exploration of mechanical stability limits in erect stance, J. Motor Behav. 21 (1989) $225-244$.

[36] M.S. Zakynthinaki, J.R. Stirling, A. López, C.A. Cordente, M. Sillero, J. Sampedro, Stochastic optimization for the calculation of the optimal critical curve from experimental data in a model of the process of regaining balance after perturbation from quiet stance, Comput. Phys. Commun. 179 (8) (2008) $562-568$.

[37] B.G. Bardy, O. Oullier, R.J. Bootsma, T.A. Stoffregen, Dynamics of human postural transitions, J. Exp. Psychol. Human Percept. Perform. 28 (2002) 499514.

[38] K. Levenberg, A method for the solution of certain non-linear problems in least squares, Q. Appl. Math. 2 (1944) $164-168$.

[39] C.M. Green, M.J. Petrou, M.L.S. Forgarty-Hover, C.G. Rolf, Injuries among judokas during competition, Scand. J. Med. Sci. Sports 17 (2007) $205-210$.

[40] H. Moksnes, M.A. Risberg, Performance-based functional evaluation of non-operative and operative treatment after anterior cruciate ligament injury, Scand. J. Med. Sci. Sports 19 (2009) 345-355.

[41] J.R. Stirling, M.S. Zakynthinaki, A model of stability and balance, in: A. Wit, F. Vaverka (Eds.), Mechanical loads of the human motor system - Injury prevention, volume 64 of Lecture notes of the ICB, Seminar, 2005, p. 39. 